\title{
表观反射率及其在植被遥感中的应用
}

池宏康 周广胜 许振柱 肖春旺 袁文平

(中国科学院植物研究所植被数量生态学重点实验室, 北京 100093)

\begin{abstract}
摘 要 由于植被遥感应用定量化和监测等的需求, 光学遥感数据的辐射校正更加受到重视。该文论述了辐射校 正, 辐射定标和大气校正的概念以及它们之间的区别及关系。特别对辐射定标的结果之一, 大气层顶表观反射率, 简称表观反射率 (Apparent reflectance)的定义、概念、计算和它在植被遥感中的应用等方面, 进行了详细的论述。 关键词 表观反射率 辐射校正 辐射定标 大气校正 植被遥感
\end{abstract}

\section{APPARENT REFLECTANCE AND ITS APPLICATIONS IN VEGETATION REMOTE SENSING}

\author{
CHI Hong-Kang ZHOU Guang-Sheng XU Zhen-Zhu XIAO Chun-Wang and YUAN Wen-Ping \\ ( Laboratory of Quantitative Vegetation Ecology, Institute of Botany, Chinese Academy of Sciences, Beijing 100093, China)
}

\begin{abstract}
Remote sensing of vegetation is developing into a highly quantitative science. However, some concepts and terms related to quantitative remote sensing are confusing for novices of this field. The goal of this paper is to clarify fuzzy concepts and promiscuous terms and to introduce methods about data processing of remote sensing information. First, concepts about radiometric correction, radiometric calibration, atmospheric correction and the differences and relationships between them were discussed. In optical remote sensing, radiometric correction includes radiometric calibration and atmospheric correction. The aim of radiometric calibration is to eliminate the errors in the sensors of the satellite, and the aim of atmospheric correction is to eliminate the effects of atmospheric interference on satellite data. In general, the first step is radiometric calibration and then atmospheric correction. Apparent reflectance is one of the results of radiometric calibration and is a very important physical variable in processing remote sensing data. The definition of apparent reflectance can be indicated with a formula. The calculation of apparent reflectance was explained step-by-step using examples with Landsat-5 and Landsat-7 satellite images. A case study was presented in order to show the importance of apparent reflectance in the calculation of NDVI (Normalized difference vegetation index). Finally, its applications in vegetation remote sensing were listed and discussed.
\end{abstract}

Key words Apparent reflectance, Radiometric correction, Radiometric calibration, Atmospheric correction, Vegetation remote sensing

最近十几年植被遥感向定量化和动态监测方向 深入发展。许多文章涉及到植被的生物量( Todd $e t$ al., 1998; Sannier et al., 2002; Foody et al., 2003)、 净初级生产力 (NPP) (Reich, 1999; Belchansky \& Douglas, 2002; Dawson et al., 2003) 的估测; 草地退 化和森林破坏等的监测 (Di Maio Mantovani \& Setzer, 1997; Tucker \& Townshend, 2000; Holm et al ., 2003; Hostert et al., 2003)。另外, 在植被遥感中经常涉及 到植被指数, 例如, 归一化差植被指数 (NDVI)。用 何种遥感数据计算才能得到较真实的植被指数? 对 于同一块森林或草地, 如何对比和监测它们在不同 时期的状况? 这些研究均需要定量化的方法。这 样, 光学遥感中原来一直存在, 但是被忽视的一些问
题被再次提出。其中, 光学遥感数据的辐射校正就 是一个典型例子。许多有关遥感的书籍和文章中, 辐射校正的概念、定义和方法等内容涉及较少, 而且 有些概念和术语时常混淆。这些都影响了遥感技术 应用水平的提高。

本文试图对辐射校正中存在的一些容易混淆的 概念和术语, 进行澄清。特别对植被遥感应用定量 化、监测, 具有特殊作用的大气层顶表观反射率( 简 称表观反射率, Apparent reflectance) 的定义, 计算及 在植被遥感中的应用等方面, 进行了详细分析和研 究, 以期对使用光学遥感数据进行各种植被遥感应 用的研究人员有一定的参考价值。 


\section{1 背 景}

1972 年美国发射了第一颗地球资源卫星, 初期 大量的遥感应用主要集中于地物 (土壤、植被和水体 等) 的识别与分类。用户得到的遥感数据( MSS、TM) 是整数的 $D N$ 值 (Digital number value)。用户可以直 接用这些原始遥感数据对各种地物进行识别和分 类。由于来自水体、植被、土壤和城市等地物的信号 很强, 传感器和大气的影响不足以妨碍到对这些地 物之间差异的探测和识别。使用 $D N$ 值数据不仅不 影响结果, 而且处理速度较快。因此, 在相当长一段 时间内, 辐射校正被忽视。随着遥感应用领域的扩 展, 资源和环境的遥感监测越来越重要, 遥感定量化 程度要求更高, 内容不再限于森林变为草原或草原 改变为农田这样质变的监测, 提出了对土地覆盖类 型, 在某一时间内无质变的监测新需求。例如, 对 一片草地,要求监测 10 或 20 年内, 它的生物量、生 产力和覆盖度等的变化情况。植被的这些生态学特 征量, 要用生物物理量, 例如, 叶面积指数 ( $L A I)$ 、光 合有效辐射 (PAR) 等来获取。这些生物物理量的差 异信息微弱, 要消除大气这样的环境影响因素,才有 可能获得。另外, 光学传感器的性能随时间变化, 引 起输出信号值的漂移。这样遥感数据之间的长时间 比较, 精度也将受到影响。原始遥感数据的 $D N$ 值 已不适宜直接用来监测这种类型的变化, 它们需要 经过辐射校正后,才能胜任遥感监测的新需求。

长期以来, 各种书刊关于辐射校正 (Radiometric correction) 的定义和内容没有统一。辐射定标（Radiometric calibration)、辐射校正和大气校正( Atmospheric correction) 使一些初步参与遥感应用的人员 感到困惑。他们不知道三者之间的区别和关系。有 的人员将辐射定标与辐射校正等同,有的则认为大 气校正是独立于辐射校正的。从光学遥感数据的获 取过程, 我们知道地物反射的辐射亮度 $L$ (Radiance) 通过大气层, 然后被卫星传感器接收, 最后转换为
$D N$ 值。理想状况下, 光学遥感传感器各波段纪录 下的辐射通量应该是地物反射的精确测量值。然 而, 误差 (噪声)在遥感数据获取过程中, 通过几种途 径混杂进来。辐射校正的目的就是消除这些误差 (噪声)。它们包括, 由传感器本身产生的内部误差 和由环境影响一大气和地形影响引起的外部误 差。内部误差一般是系统的、可以预测的, 通过卫星 发射之前的辐射定标 (Pre-flight calibration) 和运行中 的星上辐射定标 (On board calibration)、替代 (场地) 辐射定标 (Vicarious calibration) 来确定。而外部误差 在自然界是变化的、不确定的, 非系统误差。一般在 内部误差校正(即辐射定标)后, 由用户自己来消除 这种误差。在平原地区, 只进行大气校正即可消除 它, 而在山区, 除大气校正外, 有时还要进行地形辐 射校正。图 1 清楚地说明了辐射校正、辐射定标和 大气校正等之间的关系。辐射定标的目的是消除传 感器本身产生的误差。大气校正则消除大气散射、 吸收等引起的误差。对在光学遥感数据获取过程 中, 产生的一切与辐射有关的误差的校正(包括辐射 定标和大气校正) 统称辐射校正。在遥感监测等具 体应用中, 大气校正难度较大。有时只能依据遥感 影像中的暗色物体(例如湖泊)校正, 不能完全消除 大气的影响。然而, 根据辐射定标, 传感器产生的误 差则较容易消除。辐射定标的结果是给出一个联系 $D N$ 值与辐射亮度 $L$ 关系的公式和一些参数。这样 根据遥感数据中的 $D N$ 值和这些参数, 就可以计算 出相应的进入传感器的辐射亮度值和表观反射率。 遥感应用人员特别关注能反映地物性质和特征的反 射率, 因此,下面主要论述表观反射率。

\section{2 表观反射率}

\section{1 表观反射率的概念}

在文献中, 表观反射率有多个不同的提法。例 如,场景的大气层顶等价反射率( The TOA equivalent reflectance of scene)(Dinguirard \& Slater, 1999); 表观

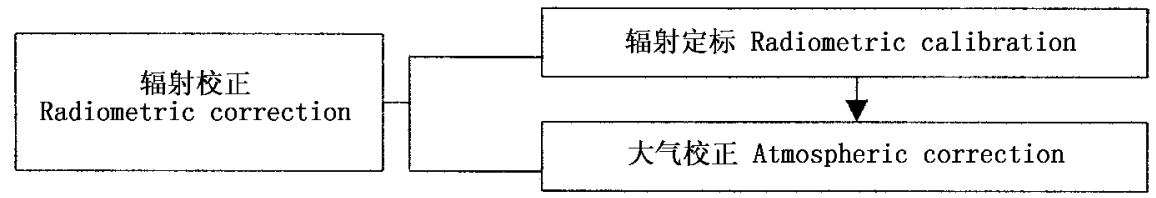

图 1 辐射校正、辐射定标和大气校正之间的区别与相互关系

Fig. 1 The difference and relationship between radiometric correction, radiometric calibration and atmospheric correction 
星上反射率 (Apparent at-satellite reflectance) (Hill \& Sturm, 1991) 星上光谱行星反照率( At-satellite spectral planetary albedos)( Markham \& Barker，1987)和等 价光谱反照率 (Equivalent spectral albedo) ( Price, 1987)等等。目前, 虽然这个物理量有不同的名称, 但是它的定义可以用 (1) 式来表达 (Roderick et al., 1996; Markham \& Barker, 1987)。

$$
\rho=\frac{\pi \cdot L \cdot D^{2}}{E S U N \cdot \operatorname{Cos} \theta}
$$

式中, $\rho$ 为大气层顶 $(T O A$ ) 表观反射率 (无量纲), $\pi$ 为常量 (球面度 $s r$ ), $L$ 为大气层顶进入卫星传感器 的光谱辐射亮度 $\left(\mathrm{W} \cdot \mathrm{m}^{-2} \cdot \mathrm{sr}^{-1} \cdot \mu \mathrm{m}^{-1}\right), D$ 为日地之 间距离 (天文单位), $\mathrm{ESUN}$ 为大气层顶的平均太阳光 谱辐照度 $\left(\mathrm{W} \cdot \mathrm{m}^{-2} \cdot \mu \mathrm{m}^{-1}\right), \theta$ 为太阳的天顶角。

以上 (1) 式成立的条件是假设在大气层顶，有 一个朗勃特 (Lanbertian) 反射面。太阳光以天顶角 $\theta$ 入射到该面。如图 2, 该表面的辐照度为 $E=E S U N$ - $\cos \theta / D^{2}$ (吕斯骅, 1981)。该表面的辐射出射度 $M$ $=\pi L$ （吕斯骅, 1981）。根据 Lanbertian 反射率定 义, 大气层顶的表观反射率 $\rho$ 等于 $M$ 和 $E$ 的比值, 即 $\rho=\frac{M}{E}=\frac{\pi L D^{2}}{E S U N \cos \theta}$ 。这就是上面 (1) 式的由来。 这里还有三点需要说明。

第一, $L$ 和 $E S U N$ 分别为光谱辐射亮度和光谱 辐照度，与波段有关，不同的波段有其相应的值。

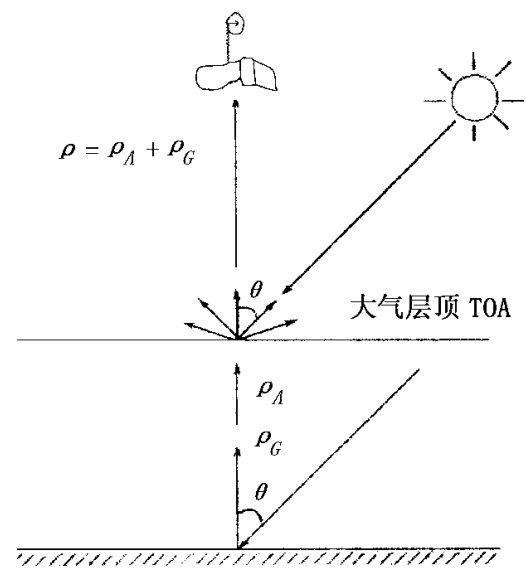

图 2 大气层顶 $(\mathrm{TOA})$ 的表观反射率 $\rho$

Fig. 2 Apparent reflectance $\rho$ at the top of atmosphere (TOA)

第二, 注意单位的一致。例如, $L$ 的单位是 $\mathrm{W} \cdot$ $\mathrm{m}^{-2} \cdot \mathrm{sr}^{-1} \cdot \mu \mathrm{m}^{-1}$ 。如果资料给出的 $L$ 为 $\mathrm{mW} \cdot \mathrm{cm}^{-2}$. $\mathrm{sr}^{-1} \cdot \mu \mathrm{m}^{-1}$, 应该换算为前者 $\left(1 \mathrm{~W} \cdot \mathrm{m}^{-2} \cdot \mathrm{sr}^{-1} \cdot \mu \mathrm{m}^{-1}\right.$ $\left.=0.1 \mathrm{~mW} \cdot \mathrm{cm}^{-2} \cdot \mathrm{sr}^{-1} \cdot \mu \mathrm{m}^{-1}\right)$ 。

第三, $L$ 是大气层顶进入卫星传感器的光谱辐 射亮度。实际上, 它是来自地物和大气辐射亮度的 总和。因此, 大气层顶的表观反射率 $\rho$ 也是地面反
射率 $\rho_{G}$ 和大气反射率 $\rho_{A}$ 的总和, 即:

$$
\rho=\rho_{G}+\rho_{A}
$$

\section{2 表观反射率的计算}

分为两个步骤。首先计算光谱辐射亮度 $L$, 然 后根据(1)式求取表观反射率 $\rho$ 。

\subsection{1 光谱辐射亮度的计算}

$L$ 是大气层顶卫星传感器接收的辐射亮度。如 前所述, 辐射定标就是确定 $L$ 与 $D N$ 的关系式, 以及 相应的定标参数增益 (Gain) 和偏移 (Bias)。由于光 学传感器器件性能的退化, 这些参数也在改变。通 过星上对传感器的辐射定标和地面的替代辐射定 标, 不断修正这些参数。地面站给用户提供卫星的 $D N$ 数据时, 这些参数也同时被提供。例如, 用户得 到一帧 2001 年 8 月 14 日, 内蒙古锡林浩特附近的 Landsat-7 ETM + 的 $D N$ 数据。在提供的文件中, 可 以查到 ETM + 1 5,7 波段的 Gain 和 Bias 的参数值 (本文主要论述 Landsat-5 和 Landsat-7 的 TM 和 ETM + 中的 $1 \sim 5,7$ 反射波段)。根据以下公式(3), 我 们就可以分别计算出各个波段每个像元的辐射亮度 $L$ 值。

$$
L=\text { Gain } \bullet D N+\text { Bias }
$$

如果, 没有定标参数 Gain 和 Bias 的资料, 某一 波段的 $L$ 可以根据 (4) 式计算。(3)和(4)式均来自 网上 ( http: //ltpwww. gsfc. gov/IAS/handbook/handbook-htmls/chapter11/chapter11.html)。

$$
\begin{aligned}
& L=\frac{L_{\text {max }}-L_{\text {min }}}{Q C A L_{\text {max }}-Q C A L_{\text {min }}} \cdot\left(Q C A L-Q C A L_{\text {min }}\right)+ \\
& L_{\text {min }}
\end{aligned}
$$

式中, $Q C A L$ 为某一像元的 $D N$ 值, 即 $Q C A L=$ $D N$ 。QCAL $L_{\max }$ 为像元可以取的最大值 255 。 $Q C A L_{\text {min }}$ 为像元可以取的最小值。如果卫星数据来自 LPGS (The level 1 product generation system), 则 $Q C A L_{\text {min }}=1$ (Landsat-7 数据属于此类型)。如果卫星数据来自美 国的 NLAPS ( National Landsat Archive Production System), 则 $Q C A L_{\text {min }}=0$ (Landsat-5 的 TM 数据属于此类 型)。

根据以上情况, 对于 Landsat-7 来说, (4) 式可以 改写为 (5) 式 $\left(Q C A L_{\text {min }}=1\right)$ 。

$$
L=\frac{L_{\max }-L_{\min }}{254} \cdot(D N-1)+L_{\text {min }}
$$

对于 Landsat-5 来说, (4) 式可以改写为 (6) 式 $\left(Q C A L_{\mathrm{MIN}}=0\right)$

$$
L=\frac{L_{\text {max }}-L_{\text {min }}}{255} \cdot D N+L_{\text {min }}
$$

$L_{\text {max }}$ 和 $L_{\text {min }}$ 分别为 $Q C A L=255$ 和 $Q C A L=1$ (或 
0 ) 时的光谱辐射亮度值。我们可以在网上 (NASA Landsat Project Science Office, 1998a)查找到 Landsat-7 $\mathrm{ETM}+$ 各个反射波段的 $L_{\text {max }}$ 和 $L_{\text {min }}$ 值(表 1 )。

Landsat-7 的 $L_{\max }$ 和 $L_{\min }$ 选取要注意两个问题。 首先, 了解卫星资料获取的时间。2000 年 7 月 1 日 之前的, 采用前面 4 列; 反之, 用后面 4 列。第二个 问题涉及到高增益 (High gain) 和低增益 (Low gain) 的参数。为了使传感器的辐射分辨率达到最大, 而 又不使其达到饱和, 根据地表类型 (非沙漠和冰面的 陆地、沙漠、冰与雪、水体、海冰、火山等 6 大类型)和 太阳高度角状况来确定采用高增益参数或是低增益 参数。一般低增益的动态范围比高增益大 1.5 倍, 因此当地表亮度较大时, 用低增益参数; 其它情况用 高增益参数。在非沙漠和冰面的陆地地表类型中, $\mathrm{ETM}+$ 的 $1 \sim 3$ 和 5.7 波段采用高增益参数, 4 波段 在太阳高度角低于 $45^{\circ}$ 时也用高增益参数, 反之则用 低增益参数。详见文献( NASA Landsat Project Science Office, 1998b)。

根据上面的两种情况, 前面提到的内蒙古锡林
浩特附近的 Landsat-7 卫星数据( 获取于 2001 年 8 月 14 日)应该采用后面 4 列参数, 而且除了 4 波段(太 阳高度角为 $54.1^{\circ}$ ) 采用低增益的 $L_{\max }$ 和 $L_{\min }$ 参数 外, 其余 $1 \sim 3$ 和 5.7 波段均采用高增益的 $L_{\max }$ 和 $L_{\min }$ 参数。

Landsat-5 发射于 1984 年 3 月。现在, 20 年过去 了, 它仍旧在轨道上正常运转。总体上看, 在整个运 行期间, 它的传感器有较好的辐射稳定性。然而, 内 部的定标器灯 (Calibrator lamps) 的亮度在逐渐增加, 特别在 1988 年以后, 这种状况更加明显。为了使 Landsat-5 的 TM 辐射定标, 达到能与 Landsat-7 的 $\mathrm{ETM}+$ 进行比较的精度。最近, TM 数据用一套新的 参数来进行辐射定标。这套参数的使用, 以 2003 年 5 月 5 日为分界线(表 2)。从 Landsat-5 发射的 1984 年 3 月 1 日至 2003 年 5 月 4 日, 这段期间获取的 TM 数据的辐射定标使用表 2 中的左边数据, 而 2003 年 5 月 5 日以后, 使用右边的数据。这样, 根据 (5) 式 和(6)式, Landsat-7 和 Landsat-5 某波段每个像元的 $D N$ 值可以转换为光谱辐射亮度 $L$ 。

表 1 Landsat-7 ETM + 各个反射波段的 $L_{\text {max }}$ 和 $L_{\min }$ 值

Table 1 The values of $L_{\max }$ and $L_{\min }$ for reflecting bands of Landsat-7 ETM $+\left(\mathrm{W} \cdot \mathrm{m}^{-2} \cdot \mathrm{sr}^{-1} \cdot \mu \mathrm{m}^{-1}\right)$

\begin{tabular}{|c|c|c|c|c|c|c|c|c|}
\hline \multirow{3}{*}{$\begin{array}{l}\text { 波段 } \\
\text { Band }\end{array}$} & \multicolumn{4}{|c|}{ 2000/07/01 之前 Before July 1, 2000} & \multicolumn{4}{|c|}{ 2000/07/01 之后 After July 1, 2000} \\
\hline & \multicolumn{2}{|c|}{ 低 Gain～Low Gain } & \multicolumn{2}{|c|}{ 高 Gain High Gain } & \multicolumn{2}{|c|}{ 低 Gain Low Gain } & \multicolumn{2}{|c|}{ 高 Gain High Gain } \\
\hline & $L_{\min }$ & $L_{\max }$ & $L_{\min }$ & $L_{\max }$ & $L_{\min }$ & $L_{\max }$ & $L_{\min }$ & $L_{\max }$ \\
\hline 1 & -6.2 & 297.5 & -6.2 & 194.3 & -6.2 & 293.7 & -6.2 & 191.6 \\
\hline 2 & -6.0 & 303.4 & -6.0 & 202.4 & -6.4 & 300.9 & -6.4 & 196.5 \\
\hline 3 & -4.5 & 235.5 & -4.5 & 158.6 & -5.0 & 234.4 & -5.0 & 152.9 \\
\hline 4 & -4.5 & 235.0 & -4.5 & 157.5 & -5.1 & 241.1 & -5.1 & 157.4 \\
\hline 5 & -1.0 & 47.70 & -1.0 & 31.76 & -1.0 & 47.57 & -1.0 & 31.06 \\
\hline 7 & -0.35 & 16.60 & -0.35 & 10.932 & -0.35 & 16.54 & -0.35 & 10.80 \\
\hline
\end{tabular}

表 2 Landsat-5 TM 各个反射波段的 $L_{\max }$ 和 $L_{\min }$ 值 Table 2 The values of $L_{\max }$ and $L_{\min }$ for reflecting bands of Landsat-5 TM $\left(\mathrm{W} \cdot \mathrm{m}^{-2} \cdot \mathrm{sr}^{-1} \cdot \mu \mathrm{m}^{-1}\right)$

\begin{tabular}{cccccc}
\hline \multirow{2}{*}{$\begin{array}{r}\text { 波段 } \\
\text { Band }\end{array}$} & $\begin{array}{c}\text { 1984/03/01 至 } 2003 / 05 / 04 \\
\text { From Mar.1, } 1984 \text { to }\end{array}$ & & \multicolumn{2}{c}{$\begin{array}{c}2003 / 05 / 05 \\
\text { Mfter May } 5,2003\end{array}$} \\
\cline { 2 - 3 } \cline { 5 - 6 } & \multicolumn{2}{c}{$L_{\min }$} & $L_{\max }$ & & \multicolumn{2}{c}{$L_{\min }$} & $L_{\max }$ \\
\hline 1 & -1.52 & 152.10 & & -1.52 & 193.0 \\
2 & -2.84 & 296.81 & & -2.84 & 365.0 \\
3 & -1.17 & 204.30 & & -1.17 & 264.0 \\
4 & -1.51 & 206.20 & & -1.51 & 221.0 \\
5 & -0.37 & 27.19 & & -0.37 & 30.2 \\
7 & -0.15 & 14.38 & -0.15 & 16.5 \\
\hline
\end{tabular}

\subsection{2 表观反射率的计算}

Landsat-7 和 Landsat-5 的 $1 \sim 5,7$ 波段的表观反 射率均用前面的公式 (1) 计算。其中, $\pi$ 是常量; 光 谱辐射亮度 $L$ 用 (5) 和 (6) 式求得; $D$ 为日地之间距 离, 根据表 3 , 可以推算全年任何一天的日地距离; $E S U N$ 为大气层顶的平均太阳光谱辐照度, 根据表 4 可查得。

$\theta$ 为太阳的天顶角, 地面站提供的头文件给出 的是太阳高度角 $\beta$, 因此, $\theta=90^{\circ}-\beta$ 。另外, 可以 用(7)式 (吕斯骅, 1981), 直接求取 $\cos \theta$ 。

$$
\operatorname{Cos} \theta=\operatorname{Sin} \phi \cdot \operatorname{Sin} \delta+\operatorname{Cos} \phi \cdot \operatorname{Cos} \delta \bullet \operatorname{Cos} h
$$


式中: $\varphi$ 为地理纬度, $\delta$ 为太阳赤纬, $h$ 为太阳 的时角。太阳赤纬是太阳光与地球赤道平面的夹 角,一年中它在 $\pm 23^{\circ} 27^{\prime}$ 之间变动, 春分和秋分时太 阳直射赤道 $\delta=0$, 夏至时太阳直射北回归线 $\delta=$ $23^{\circ} 27^{\prime}$, 冬至时太阳直射南回归线 $\delta=-23^{\circ} 27^{\prime}$ 。一 年内 $\delta$ 的变化值可以查天文年历。太阳时角以地 方时 12:00 的时角 $h$ 为 $0,6: 00$ 为 $-\pi / 2,18: 00$ 为 $+\pi / 2$ 。

\subsection{3 表观反射率的计算实例}

下面以 2001 年 8 月 14 日内蒙古锡林浩特附近 的 Landsat-7 卫星 $D N$ 数据(表 5(b)) 为例, 计算 5 种 地物在波段 ETM3 和 ETM4 中的光谱辐射亮度 $L$ 和 表观反射率 $\rho$ 。计算所需要的参数见表 $5(\mathrm{a})$, 特别 要注意 ETM4 的 $L_{\max }$ 和 $L_{\min }$ 的选取和天顶角 $\theta$ 的取 值。具体的计算按照前面介绍的步骤进行。计算结 果见表 5(b)。

表 3 随时间变化的日地距离 (天文单位)

Table 3 Earth-Sun distance at different time (Astronomical units)

\begin{tabular}{cccccccccc}
\hline $\begin{array}{c}\text { 日数 } \\
\text { Day }\end{array}$ & $\begin{array}{c}\text { 距离 } \\
\text { Distance }\end{array}$ & $\begin{array}{c}\text { 日数 } \\
\text { Day }\end{array}$ & $\begin{array}{c}\text { 距离 } \\
\text { Distance }\end{array}$ & $\begin{array}{c}\text { 日数 } \\
\text { Day }\end{array}$ & $\begin{array}{c}\text { 距离 } \\
\text { Distance }\end{array}$ & $\begin{array}{c}\text { 日数 } \\
\text { Day }\end{array}$ & $\begin{array}{c}\text { 距离 } \\
\text { Distance }\end{array}$ & $\begin{array}{c}\text { 日数 } \\
\text { Day }\end{array}$ & $\begin{array}{c}\text { 距离 } \\
\text { Distance }\end{array}$ \\
\hline 1 & 0.9832 & 74 & 0.9945 & 152 & 1.0140 & 227 & 1.0128 & 305 & 0.9925 \\
15 & 0.9836 & 91 & 0.9993 & 166 & 1.0158 & 242 & 1.0092 & 319 & 0.9892 \\
32 & 0.9853 & 106 & 1.0033 & 182 & 1.0167 & 258 & 1.0057 & 335 & 0.9860 \\
46 & 0.9878 & 121 & 1.0076 & 196 & 1.0165 & 274 & 1.0011 & 349 & 0.9843 \\
60 & 0.9909 & 135 & 1.0109 & 213 & 1.0149 & 288 & 0.9972 & 365 & 0.9830 \\
\hline
\end{tabular}

表 4 Landsat-7 和 Landsat-5 的大气层顶平均太阳光谱辐照度

Table 4 Mean solar spectral irradiance at the atmospheric top for Landsat-7 and Landsat-5 $\left(\mathrm{W} \cdot \mathrm{m}^{-2} \cdot \mu \mathrm{m}^{-1}\right)$

\begin{tabular}{cccccccc}
\hline 波段 Band & 1 & 2 & 3 & 4 & 5 & 7 \\
\hline Landsat-7 ESUN & 1969.00 & 1840.00 & 1551.00 & 1044.00 & 225.70 & 82.07 \\
Landsat-5 ESUN & 1957.00 & 1826.00 & 1554.00 & 1036.00 & 215.00 & 80.67 \\
\hline
\end{tabular}

表 55 种地物在波段 ETM3 和 ETM4 中的光谱辐射亮度和表观反射率计算实例

Table 5 Case study on spectral radiance and apparent reflectance of five targets in ETM3 and ETM4

(a) 计算 ETM3 和 ETM4 中的光谱辐射亮度和表观反射率所需要的参数 The parameters in computing spectral radiance and apparent reflectance for ETM3 and ETM4

\begin{tabular}{ccccccc}
\hline $\begin{array}{c}\text { 波段 Band } \\
\text { ETM }+\end{array}$ & $\begin{array}{c}L_{\max } \\
\left(\mathrm{W} \cdot \mathrm{m}^{-2} \cdot \mathrm{sr}^{-1} \cdot \mu \mathrm{m}^{-}\left(\mathrm{W} \cdot \mathrm{m}^{-2} \cdot \mathrm{sr}^{-1} \cdot \mu \mathrm{m}^{-1}\right)\right.\end{array}$ & $\begin{array}{c}\pi \\
(\mathrm{sr})\end{array}$ & $D$ & $\begin{array}{c}\text { ESUN } \\
\mathrm{W} \cdot \mathrm{m}^{-2} \cdot \mu \mathrm{m}^{-1}\end{array}$ \\
\hline 3 & -5.0 & 152.9 & 3.14159 & 1.01295 & 1551.00 & $35.9^{\circ}$ \\
4 & -5.1 & 241.1 & 3.14159 & 1.01295 & 1044.00 & $35.9^{\circ}$ \\
\hline
\end{tabular}

(b) 5 种地物在波段 ETM3 和 ETM4 中的 $D N$ 值和计算得到的 $L 、 \rho$ 和 $N D V I$ 值 Values of $D N$ and $L, \rho$ and $N D V I$ derived from calculation for five targets in ETM3 and ETM4

\begin{tabular}{|c|c|c|c|c|c|c|c|c|c|}
\hline \multirow{2}{*}{$\begin{array}{l}\text { 地物 } \\
\text { Target }\end{array}$} & \multicolumn{2}{|c|}{$D N$} & \multicolumn{2}{|c|}{$L$} & \multicolumn{2}{|c|}{$\rho$} & \multicolumn{3}{|c|}{$N D V I$} \\
\hline & ETM3 & ETM4 & L3 & L4 & $\rho 3$ & $\rho 4$ & $D N$ & $L$ & $\rho$ \\
\hline 林地 Woodland & 45 & 95 & 22.353 & 86.013 & 0.0574 & 0.3279 & 0.3571 & 0.5875 & 0.7022 \\
\hline 草地 Grassland & 96 & 74 & 54.057 & 65.658 & 0.1387 & 0.2503 & -0.1294 & 0.0969 & 0.2869 \\
\hline 农地 Farmland & 52 & 121 & 26.704 & 111.215 & 0.0685 & 0.4239 & 0.3988 & 0.6128 & 0.7217 \\
\hline 裸沙 Sand & 170 & 104 & 100.059 & 94.737 & 0.2567 & 0.3611 & -0.2409 & -0.0273 & 0.1690 \\
\hline 水体 Water & 55 & 18 & 28.569 & 11.378 & 0.0733 & 0.0434 & -0.5068 & -0.4304 & -0.2565 \\
\hline
\end{tabular}

3 表观反射率在植被遥感中的应用

\section{1 构建植被指数}

植被指数 $(V I)$ 与植被的生物量、净初级生产力 $(N P P)$ 、覆盖度, 以及冠层的叶面积指数 $(L A I)$ 等, 都 有较好的相关性。许多遥感模型和以遥感信息为驱
动变量的生态学模型都需要植被指数的参与。卫星 的 $D N$ 值、辐射亮度值 $L$ 和表观反射率 $\rho$ 均可以计 算植被指数。以归一化差植被指数 ( NDVI) 为例, $N D V I=(N I R-R) /(N I R+R), N I R$ 和 $R$ 分别为近 红外和红光, 它们计算的结果见表 5(b)。显然, 三 者用同一公式计算出了不同的 NDVI 数值。哪种 
$N D V I$ 数值接近地物的实际情况? 从理论上分析, $D N$ 未经过任何校正,包括辐射定标校正,而且只是 进入传感器中的辐射能的一种数字转换形式, 不能 本质地反映地物的辐射特性。 $L$ 和 $\rho$ 都经过了辐射 定标校正，但是，当 $\rho$ 再经过大气校正后，它就是 地物的反射率, 能本质地反映地物的辐射特性。由 它构成的 $N D V I$ 植被指数最接近地物的 $N D V I$ 。以表 5(b) 中的草地和裸沙为例。尽管草地为退化草地, 在地面它的 $N D V I$ 值可以达到 0.3 左右。然而, $L$ 的 $N D V I$ 值为 0.096 , 在地面这是裸地的 NDVI 值。 $D N$ 的 $N D V I$ 值更离谱, 为 -0.1294 , 这是地面水体 的 $N D V I$ 数值。 $\rho$ 的 $N D V I$ 值为 0.2869 , 最接近地面 退化草地的 NDVI 值。在裸沙一行中, 只有 $\rho$ 的 $N D V I$ 值 (0.1690) 接近裸沙的实际情况, $L$ 和 $D N$ 的 值均为负数, 是地面水体的 $N D V I$ 数值范围。以上 的分析和计算结果说明只有表观反射率 $\rho$ 构成的 植被指数能较好地反映地物的情况。这也是辐射定 标将卫星的 $D N$ 值转换为表观反射率 $\rho$ 的原因之

\section{2 遥感模型中的变量}

许多植被遥感应用要求用地物的光谱反射率与 生物物理量建立遥感模型。例如, SAIL 模型。

\section{3 大气校正}

在定量化植被遥感应用中, 大气影响是主要误 差来源之一, 而且消除这种影响很困难。

一般来说,要完成大气校正, 必须先进行辐射定 标。表观反射率是辐射定标的结果之一。因此,可 以认为表观反射率计算是大气校正的前期准备。前 面的(2)式, $\rho=\rho_{G}+\rho_{A}$ 表明表观反射率 $\rho$ 是地物反 射率 $\rho_{G}$ 和大气反射率 $\rho_{A}$ 的和。大气校正就是消除 $\rho_{A}$ 。有时, 由于种种原因不能用大气传输模型进行 大气校正, 可以用表观反射率进行部分简单校正。 例如, 根据遥感影像中的暗色地物, 用直方图最小值 方法校正(彭望禄，1991)。这样可以尽量减少 $\rho_{A}$, 即大气的影响。“裸沙土壤线”大气校正方法 ( Chi, 2003 ), 可以实用于干旱和半干旱地区 NOAA AVHRR 通道 1 $(R)$ 和通道 2( NIR ) 的大气校正, 即去 除 (2) 式中大气的反射率 $\rho_{A}$ 。但是, 使用的卫星数 据不是 $D N$ 值, 必须是表观反射率 $\rho$ 。

\section{4 不同时期遥感数据的比较}

在植被遥感监测中, 经常遇到不同时期遥感数 据的比较。这类遥感数据的比较, 包括同一个传感 器, 不同时间遥感数据的比较和不同传感器遥感数 据的比较。长期监测同一植被或地区，既是使用同
一个传感器, 由于光学传感器的性能随时间变化, 会 引起输出信号值的漂移。要消除这种影响, 必须进 行辐射定标, 将 $D N$ 值换算为表观反射率, 然后才能 进行不同时间遥感数据之间的比较。然而, 对于不 同传感器遥感数据的比较, 还要面对传感器之间的 波段、光谱分辨率和空间分辨率的差异等问题。当 然, 在实际遥感应用中为了尽量减少传感器之间的 差异,一般选择相同卫星系列传感器的遥感数据进 行比较, 例如, Landsat-5 的 TM 和 Landsat-7 的 ETM + 。这两个传感器的 $1 \sim 5$ 和 7 反射波段相同, 而且 地面分辨率也相同。只要将它们的 $D N$ 值分别换算 为表观反射率, 就可以相互比较。

\section{4 讨 论}

以上主要以 Landsat -5 和 Landsat-7 为例, 论述了 表观反射率的定义、概念、计算方法和在植被遥感中 的应用。以上的计算方法原理, 同样适用于其它卫 星, 例如 SPOT、NOAA 等传感器的遥感数据表观反 射率。

Landsat-5 发射于 1984 年 3 月 1 日, 至今已运行 20 年。我国卫星地面站建立于 20 世纪 80 年代中 期, 也应接收和存档了近 20 年的 Landsat-5 的数据。 美国 2003 年发布的 Landsat-5 TM 数据新的辐射定标 参数(表 2), 又为其早期数据的应用奠定了基础。 通过表观反射率, 可以在近 20 年的时间尺度上, 对 我国的植被资源和生态环境进行动态监测, 发掘 Landsat -5 遥感数据的潜在价值。

如前所述, 卫星光学传感器的性能随时间变化, 它的辐射定标参数也会改变。在计算表观反射率 时, 应上网查看是否有变化, 以新的定标参数为准。

\section{参 考 文 献}

Belchansky GI, Douglas DC (2002). Integrating remotely sensed data with an ecosystem model to estimate net primary productivity in East Asia. Remote Sensing of Environment, 81, 58-66.

Chi HK (2003) . Practical atmospheric correction of NOAA-AVHRR data using the bare-sand soil line method. International Journal of Remote Sensing, 24, 3369 - 3379.

Dawson TP, North PRJ, Plummer SE (2003). Forest ecosystem chlorophyll content: implications for remotely sensed estimates of net primary productivity. International Journal of Remote Sensing, 24, 611-617.

Di Maio Mantovani AC, Setzer AW (1997). Deforestation detection in the Amazon with an AVHRR-based system. International Journal of Remote Sensing, 18, 273-286.

Dinguirard M, Slater PN (1999). Calibration of space-multispectral imaging sensors: a review. Remote Sensing of Environment, 68 , $194-205$. 
Foody GM, Boyd DS, Cutler MEJ (2003). Predictive relations of tropical forest biomass from Landsat TM data and their transferability between regions. Remote Sensing of Environment, 85, 463 -474 .

Hill J, Sturm B (1991). Radiometric correction of multitemporal Thematic Mapper data for use in agricultural land-cover classification and vegetation monitoring. International Journal of Remote Sensing, 12, 1471 - 1491 .

Holm AM, Shane H, Cridland W, Roderick ML (2003). The use of time-integrated NOAA NDVI data and rainfall to assess landscape degradation in the arid shrubland of Western Australia. Remote Sensing of Environment, 85, 145 - 158 .

Hostert P, Röder A, Hill J, Udelhoven T, Tsiourlis G (2003). Retrospective studies of grazing-induced land degradation: a case study in central Crete, Greece. International Journal of Remote Sensing, 24, 4019 - 4034 .

Lü SH (吕斯骅) (1981). Physical Foundation of Remote Sensing (遥感的物理基础). Commercial Press, Beijing, 206-234. (in Chinese)

Markham BL, Barker JL (1987). Thematic Mapper bandpass solar exoatmospheric irradiances. International Journal of Remote Sensing, 8, $513-523$.

Peng WL (彭望禄) (1991). Computer Processing of Remote Sens- ing Data and Geographical Information System (遥感数据的计 算机处理与地理信息系统). Beijing Normal University Press, Beijing, 1 - 390. (in Chinese)

Price JC (1987) . Calibration of satellite radiometers and the comparison of vegetation indices. Remote Sensing of Environment, $21,15-27$.

Reich PB (1999). An approach to spatially-distributed modeling of net primary production (NPP) at the landscape scale and its application in validation of EOS NPP products. Remote Sensing of Environment, 70, 69-81.

Roderick M, Smith R, Lodwick G (1996). Calibrating long-term AVHRR-derived NDVI imagery. Remote Sensing of Environment, $58,1-12$.

Sannier CAD, Taylor JC, Plessis WD (2002). Real-time monitoring of vegetation biomass with NOAA-AVHRR in Etosha National Park, Namibia, for fire risk assessment. International Journal of Remote Sensing, 23, 71-89.

Todd SW, Hoffer RM, Milchunas DG (1998). Biomass estimation on grazed and ungrazed rangelands using spectral indices. International Journal of Remote Sensing, 19, 427 - 438.

Tucker CJ, Townshend JRG (2000) . Strategies for monitoring tropical deforestation using satellite data. International Journal of Remote Sensing, 21, $1461-1472$. 